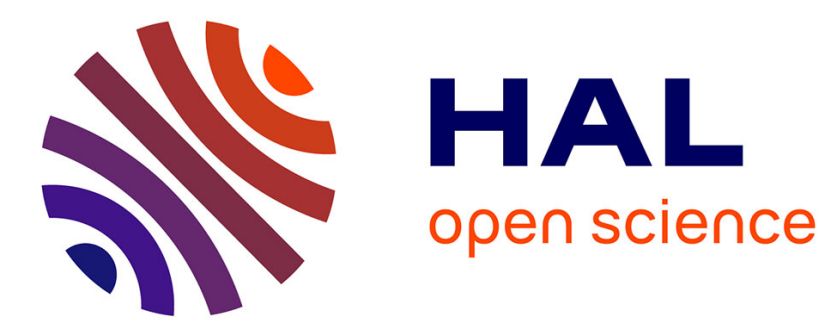

\title{
Démonétisation en Russie: un arbitrage favorable au maintien de l'emploi
}

Sophie Brana, Mathilde Maurel

\section{To cite this version:}

Sophie Brana, Mathilde Maurel. Démonétisation en Russie: un arbitrage favorable au maintien de l'emploi. Revue Economique, 2001, 52 (4), pp.841-859. hal-01016944

\section{HAL Id: hal-01016944 \\ https://hal-sciencespo.archives-ouvertes.fr/hal-01016944}

Submitted on 1 Jul 2014

HAL is a multi-disciplinary open access archive for the deposit and dissemination of scientific research documents, whether they are published or not. The documents may come from teaching and research institutions in France or abroad, or from public or private research centers.
L'archive ouverte pluridisciplinaire HAL, est destinée au dépôt et à la diffusion de documents scientifiques de niveau recherche, publiés ou non, émanant des établissements d'enseignement et de recherche français ou étrangers, des laboratoires publics ou privés. 


\title{
Persée
}

http://www.persee.fr

\section{Démonétisation en Russie. Un arbitrage favorable au maintien de l'emploi}

\author{
Mathilde Maurel;Sophie Brana \\ Revue économique, Année 2001, Volume 52, Numéro 4 \\ p. $841-859$
}

Voir l'article en ligne

\section{Avertissement}

L'éditeur du site " PERSEE » - le Ministère de la jeunesse, de l'éducation nationale et de la recherche, Direction de l'enseignement supérieur, Sous-direction des bibliothèques et de la documentation - détient la propriété intellectuelle et les droits d'exploitation. A ce titre il est titulaire des droits d'auteur et du droit sui generis du producteur de bases de données sur ce site conformément à la loi n`98-536 du 1 er juillet 1998 relative aux bases de données.

Les oeuvres reproduites sur le site «PERSEE » sont protégées par les dispositions générales du Code de la propriété intellectuelle.

Droits et devoirs des utilisateurs

Pour un usage strictement privé, la simple reproduction du contenu de ce site est libre.

Pour un usage scientifique ou pédagogique, à des fins de recherches, d'enseignement ou de communication excluant toute exploitation commerciale, la reproduction et la communication au public du contenu de ce site sont autorisées, sous réserve que celles-ci servent d'illustration, ne soient pas substantielles et ne soient pas expressément limitées (plans ou photographies). La mention Le Ministère de la jeunesse, de l'éducation nationale et de la recherche, Direction de l'enseignement supérieur, Sous-direction des bibliothèques et de la documentation sur chaque reproduction tirée du site est obligatoire ainsi que le nom de la revue et- lorsqu'ils sont indiqués - le nom de l'auteur et la référence du document reproduit.

Toute autre reproduction ou communication au public, intégrale ou substantielle du contenu de ce site, par quelque procédé que ce soit, de l'éditeur original de l'oeuvre, de l'auteur et de ses ayants droit.

La reproduction et l'exploitation des photographies et des plans, y compris à des fins commerciales, doivent être autorisés par l'éditeur du site, Le Ministère de la jeunesse, de l'éducation nationale et de la recherche, Direction de l'enseignement supérieur, Sous-direction des bibliothèques et de la documentation (voir http://www.sup.adc.education.fr/bib/ ). La source et les crédits devront toujours être mentionnés. 


\title{
Démonétisation en Russie
}

\section{Un arbitrage favorable au maintien de l'emploi}

\author{
Sophie Brana* \\ Mathilde Maurel $^{* *}$
}

\begin{abstract}
La démonétisation de l'économie russe est le produit d'une politique monétaire restrictive conjuguée au maintien de la contrainte budgétaire douce. L'État, qui autorise les arriérés fiscaux ou l'acquittement des impôts en nature, et les entreprises, qui accumulent des arriérés salariaux, arbitrent à court terme en faveur du maintien de l'emploi dans des entreprises qui diffèrent la restructuration. Le chômage est corrélé positivement à long terme aux arriérés fiscaux et salariaux, mais à court terme l'augmentation de ces derniers influence positivement l'évolution de l'emploi.
\end{abstract}

\section{RUSSIAN ARREARS: A TRADE-OF IN FAVOUR OF EMPLOYMENT}

The increase in non-monetary payments in Russia is the output of both a tight monetary policy and the persistence of the soft budget constraint. The State, by allowing fiscal arrears and in-kind payments, and the enterprises, by using wage arrears as a way of accommodating the budget constraint, make a short term arbitrage which favours the level of employment and further delay in restructuring. Unemployment is positively correlated in the long run with fiscal and wage arrears, but in the short run, any increase in the latter has a positive impact on the fluctuation of employment.

Classification JEL : E5 P2

\section{INTRODUCTION}

Le concept de démonétisation de l'économie russe renvoie à la généralisation de monnaies privées, qui sont les transactions sous forme de troc, les veksels, les zachety, ou les différents arriérés. La croissance régulière des arriérés de paiement, qui connaît une accélération depuis 1996, touche l'ensemble des agents de l'économie : l'État vis-à-vis de ses salariés ou fournisseurs, les entreprises visà-vis des banques, des salariés, des administrations publiques et des autres

\footnotetext{
* LARefi, Université Montesquieu-Bordeaux IV, avenue Léon-Duguit, 33608 Pessac. mail : brana@montesquieu.u-bordeaux.fr

** ROSES (CNRS), CEPR, Maison des Sciences économiques, 106-112 boulevard de l'Hôpital, 75013 Paris. mail : Maurelm@univ-paris1.fr

Nous remercions Ariane Tichit pour ses commentaires précieux qui ont permis d'améliorer une version antérieure de cet article.
} 
entreprises. Cette croissance des arriérés de paiement en Russie est surprenante à au moins deux égards. Tout d'abord, elle est continue, malgré de nombreuses amnisties fiscales (octobre 1993, janvier 1996, mars 1997), des accords de compensation de dettes (mars 1994, décembre 1994, avril 1996), etc., et ce, quelle que soit la situation macroéconomique sous-jacente. En outre, si le crédit interentreprises est fréquent dans de nombreux pays (à cet égard, son volume en Russie n'apparaît pas démesuré ${ }^{1}$ ), l'importance croissante des arriérés fiscaux et le développement des arriérés de salaires apparaissent plus spécifiques à la Russie $^{2}$. C'est à l'explication de ces deux phénomènes que s'attache plus particulièrement ce travail.

Deux interprétations de la démonétisation de l'économie russe sont possibles. Pour certains auteurs, le développement des transactions non monétaires et de la quasi-monnaie permet de résister à la menace de désindustrialisation que constitue le caractère restrictif de la politique monétaire. Pour d'autres, au contraire, le régime de contrainte budgétaire douce n'a pas été entamé par la politique monétaire restrictive et l'économie virtuelle, dont l'existence repose sur le développement de monnaies privées concurrentes, permet aux entreprises non viables de survivre, rendant inefficaces à la fois les politiques monétaire et fiscale.

Cet article propose une formalisation simple de l'alternative ci-dessus, qui est illustrée par l'analyse des données empiriques. La section 1 propose un modèle liant les principaux indicateurs macroéconomiques et la croissance des arriérés. Le modèle est ensuite testé à partir des données du Russian Economic Trend et du Russian Economic Barometer. La dernière section expose les conclusions de politique économique. Les «bons » indicateurs économiques affichés par les pouvoirs publics sont en grande partie «virtuels ». Le gouvernement accepte que se développent les différentes formes de paiement non monétaires, voire y contribue, car ils permettent aux entreprises de soutenir la production et l'emploi en dépit d'une politique monétaire restrictive menée depuis le début des réformes. La démonétisation permet en outre au gouvernement de respecter, en façade, les injonctions du FMI en matière de dépenses et de déficits publics.

\section{LES ARRIÉRÉS AU SECOURS DES INDICATEURS ÉCONOMIQUES}

Le durcissement, à partir de 1994, sous le contrôle des grands organismes financiers internationaux, de la politique économique de stabilisation a provoqué une croissance continue des arriérés, notamment dans le secteur des entreprises,

1. Pour Schaffer [1998], les arriérés interentreprises dans les économies en transition n'excèdent pas les niveaux observés dans les économies occidentales. Ce sont les crédits commerciaux, nécessaires à l'activité commerciale. Cependant, on peut objecter qu'une partie de ces arriérés n'étant jamais remboursée, il se produit en Russie un transfert de valeur ajoutée entre entreprises (et pas seulement de liquidités comme dans les économies occidentales).

2. En 1998, 55,2\% des arriérés sont interentreprises, 6,6\% sont des arriérés de salaire et $38,2 \%$, des arriérés fiscaux (source : Russian Economic Trend). 
en plus du développement des différentes formes de monnaies privées et du recours croissant au troc (tableau 1).

Tableau 1. Principaux indicateurs macroéconomiques

\begin{tabular}{l|r|r|r|r|r|r|c}
\hline & 1992 & 1993 & 1994 & 1995 & 1996 & 1997 & 1998 \\
\hline $\begin{array}{l}\text { Taux de croissance réel } \\
\text { de l'économie (\%) }\end{array}$ & $-14,5$ & $-8,7$ & $-12,7$ & $-4,1$ & $-3,5$ & 0,8 & $-4,6$ \\
\hline $\begin{array}{l}\text { Taux de croissance réel de la } \\
\text { production industrielle (\%) }\end{array}$ & -18 & $-14,1$ & $-20,9$ & $-3,3$ & -4 & 2 & $-5,2$ \\
\hline Taux de chômage BIr (\%) & 4,8 & 5,3 & 7,1 & 8,3 & 9,2 & 7,8 & 12,4 \\
\hline Taux officiel de chômage & & & & 2,8 & 3,5 & 3,1 & 2,6 \\
\hline Inflation annuelle (\%) & 2506,1 & 840 & 204 & 128,6 & 21,8 & 10,9 & 84,5 \\
\hline Déficit budgétaire (\% du PIB) & 4,1 & 7,4 & 9 & 5,4 & 7,9 & 7 & 5 \\
\hline Troc en \% des ventes* & 5,9 & 8,7 & 17 & 21,8 & 35 & 42,2 & 50,8 \\
\hline $\begin{array}{l}\text { Arriérés fiscaux en \% des } \\
\text { ventes annuelles }\end{array}$ & & 0 & 6,8 & 13,5 & 27,5 & 36,8 & 42,3 \\
\hline $\begin{array}{l}\text { Arriérés de salaires en \% } \\
\text { des ventes annuelles }\end{array}$ & & 0 & 2,2 & 2,5 & 4,7 & 4,6 & 5,4 \\
\hline $\begin{array}{l}\text { Arriérés interentreprises } \\
\text { en \% des ventes annuelles }\end{array}$ & & 12,2 & 11 & 22 & 33,3 & 40 & 45,8 \\
\hline
\end{tabular}

* Secteur de l'industrie.

** Secteurs de l'industrie, de la construction, du transport et agricole.

Sources : Rapport sur la transition de la BERD [1999], Russian Economic Trend, Russian Economic Barometer.

La politique de stabilisation s'est notamment traduite par un durcissement important de la politique monétaire - les taux de refinancement de la Banque centrale sont devenus durablement positifs en termes réels à partir de 1995 -, par des politiques de réduction des dépenses et des déficits publics, ainsi que par l'adoption d'un régime de taux de change fixe.

Le caractère brusquement restrictif de la politique économique aurait dû se traduire, parallèlement à la réduction du taux d'inflation, par une diminution de la production, par des fermetures d'entreprises et par une croissance importante du chômage. Or, on constate sur la période une réduction modérée de la production, une faible baisse de l'emploi et très peu de fermetures d'entreprises. Une des explications tient au développement de l'économie non monétaire, virtuelle, qui permet la survie d'une économie non rentable.

\section{Le développement de l'économie virtuelle}

Les entreprises résistent à la politique monétaire restrictive et au durcissement de la politique budgétaire en développant des monnaies privées et en laissant s'accumuler les arriérés de paiement, notamment fiscaux. L'État fait partie intégrante de ce processus qui lui permet à la fois d'afficher des indicateurs économiques politiquement et socialement soutenables et de respecter les contraintes budgétaires fixées par le FMI. La Russie n'a quasiment plus recours au seigneuriage et il n'y a pas de spirale inflationniste : l'inflation annuelle est tombée en dessous de $10 \%$ au début de 1998. Cependant, cette apparente bonne 
performance s'explique de deux manières : d'abord, le déficit, qui représente $5 \%$ du PIB en 1998, est financé à partir de 1995 par les GKO, qui substituent à l'émission monétaire la dette domestique ${ }^{1}$; ensuite (et surtout), la séquestration des sommes dues, c'est-à-dire l'accumulation d'arriérés gouvernementaux, représente environ $11 \%$ du PIB en 1996. La réduction du financement monétaire du déficit nécessite un effort fiscal et une diminution des dépenses publiques. Ce scénario ne s'est pas produit en Russie : l'État continue de financer un train de vie qui excède ses capacités de paiement, multipliant ainsi les arriérés.

Aussi, si l'on veut mesurer l'impact de la politique monétaire restrictive sur l'économie, il faut proposer deux mesures du degré de monétisation de l'économie. La première, traditionnelle, est simplement égale au rapport de l'agrégat monétaire M2 sur le PIB, la seconde au rapport de la somme de M2, des paiements dus et des substituts monétaires sur le PIB. Commander et Mumssen [1998] ont calculé la vitesse de circulation de la monnaie à partir de ces deux indicateurs : elle est respectivement égale à 7 (soit : $\mathrm{M} 2 / \mathrm{PIB}=14,3 \%$ ) et 2 (soit : $(\mathrm{M} 2+$ paiements dus + substituts monétaires $) / \mathrm{PIB}=50 \%)$ en $1998^{2}$. Cette augmentation du taux de liquidité effectif de l'économie soit évite un processus de désindustrialisation massive, soit rend illusoire le caractère restrictif de la politique monétaire en permettant à un certain nombre d'entreprises de survivre (indûment) et/ou de différer les restructurations. L'alternative peut être discutée très simplement à partir de l'équation quantitative de la monnaie :

$$
\mathrm{M}=\mathrm{P} * \mathrm{Y} / \mathrm{V}
$$

avec $\mathrm{P}$ le niveau général des prix, $\mathrm{M}$ la somme de $\mathrm{M} 2$, des paiements dus et des substituts monétaires, V la vitesse de circulation de la monnaie (que l'on suppose constante) et Y le PIB. En différenciant les termes de l'équation (1), on obtient :

$$
\dot{\mathrm{M}} / \mathrm{M}=\dot{\mathrm{P}} / \mathrm{P}+\dot{\mathrm{Y}} / \mathrm{Y}
$$

avec $\dot{\mathrm{P}} / \mathrm{P}$ le taux d'inflation, $\dot{\mathrm{M}} / \mathrm{M}$ l'augmentation de la quantité de monnaie en circulation et $\dot{Y} / Y$ le taux de croissance de l'économie.

À inflation donnée, une croissance de la masse monétaire majorée par la prise en compte de la quasi-monnaie doit se traduire par une plus forte croissance ; mais, à croissance réelle constante, elle se traduira par un plus haut niveau d'inflation cachée. Les deux interprétations renvoient à l'opposition évoquée plus haut entre partisans d'une politique monétaire stricte et partisans d'un relâchement de la politique monétariste si celle-ci, en durcissant la contrainte de liquidité, contribue à désindustrialiser l'économie. Les faits empiriques que nous mettons en évidence dans cet article suggèrent que l'augmentation de la masse monétaire ne contribue pas à maintenir des activités réellement productives, mais à développer une inflation cachée, ou virtuelle, supérieure à l'inflation officielle. Elle permet aux entreprises de valoriser leur production mais également de maintenir (à court terme) le niveau de la production. 1998.

1. Les intérêts sur les GKO représentent plus de la moitié des dépenses budgétaires en

2. En 1996, ces deux ratio sont égaux à 8 (soit : $\mathrm{M} 2 / \mathrm{PIB}=12,5 \%$ ) et 3 (soit : $(\mathrm{M} 2+$ paiements dus + substituts monétaires)/PIB $=33 \%)$. 
La notion d'inflation cachée n'est pas nouvelle en Russie : dans l'économie planifiée, elle était étroitement associée à l'existence de pénuries, c'est-à-dire que les biens n'étaient pas disponibles aux prix affichés. Aujourd'hui, selon les prix mondiaux, les entreprises russes ne sont pas compétitives. Elles organisent donc des échanges de troc dans lesquels les prix sont supérieurs aux prix monétaires. Mais l'inflation cachée repose en dernière instance sur une injection nette de crédit dans le secteur des entreprises. Cette création nette de crédits est possible par le développement des arriérés fiscaux, des arriérés de salaires et par le paiement en nature des impôts et des salaires. Elle est inflationniste dans la mesure où elle contribue à maintenir un régime de contrainte budgétaire douce et qu'elle ne correspond à aucune perspective de croissance réelle.

\section{L'arbitrage inflation/ chômage}

Dans une économie de marché traditionnelle, la politique monétaire sert à créer une inflation surprise qui maintient à court terme l'activité. À long terme cependant, les agents révisent leurs anticipations d'inflation à la hausse, et l'activité redescend au niveau correspondant au chômage naturel. En Russie, l'inflation cachée correspond à une quasi-création monétaire sous forme d'accumulation d'arriérés fiscaux, salariaux et de paiements en nature, qui permet de maintenir l'activité à un niveau supérieur à son niveau d'équilibre, et de maintenir les taux de liquidité et d'inflation officiels à un niveau inférieur au niveau réel, conformément aux objectifs affichés pour la politique monétaire.

Graphique 1.

La relation inflation (démonétisation)/ chômage

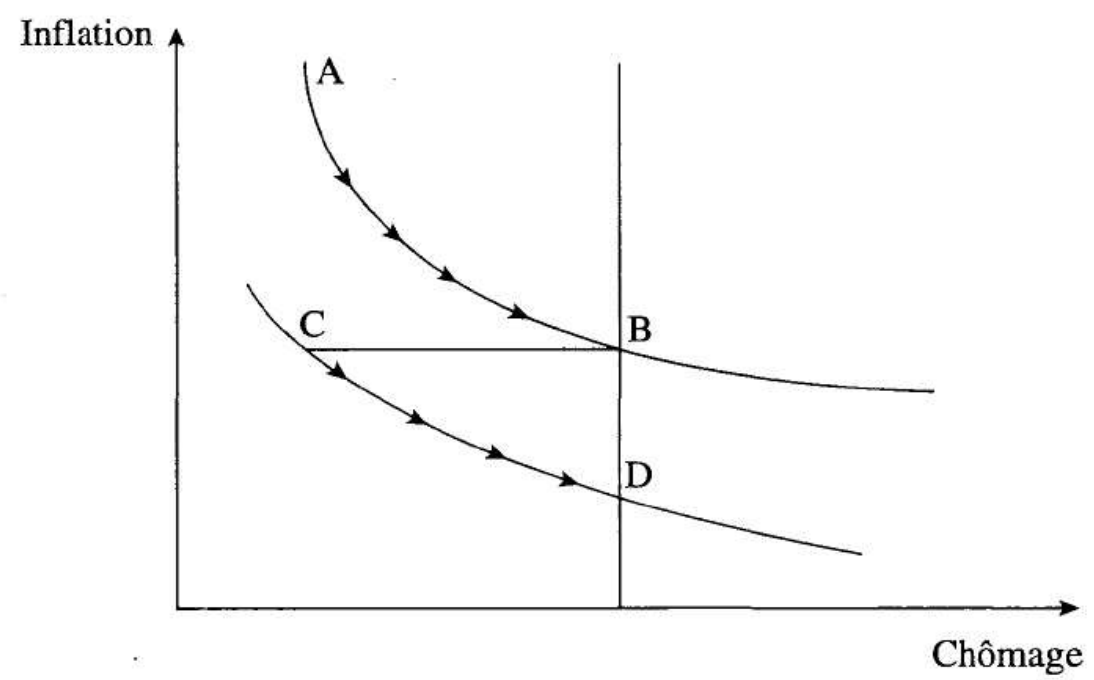

Lorsque la politique monétaire devient plus restrictive, la baisse de l'inflation se traduit par une hausse des coûts de production, à travers l'augmentation du salaire réel, ce qui provoque une diminution de l'activité et de l'emploi. L'économie tend à se déplacer le long de la courbe de Phillips de A vers B. Aux prix . de marché, un certain nombre d'entreprises détruisent de la valeur (Gaddy et Ickes [1999]), ce qui doit conduire à des restructurations et à une hausse du chômage. Cette situation était masquée dans l'ancien régime en raison de l'ar- 
bitraire des prix. Aujourd'hui, c'est la démonétisation de l'économie qui permet à ces firmes de survivre. Les entreprises réagissent au durcissement de la politique monétaire de deux façons. En premier lieu, elles maintiennent leurs prix de production « au-dessus des prix du marché » en ayant recours au troc (Ericsson et Ickes [1999]). Ce dernier utilise des prix non monétaires, supérieurs aux prix de marché, qui créent des revenus « virtuels ». En second lieu, la démonétisation permet aux entreprises de réduire leurs coûts de production. Elles accumulent ainsi des impayés dans les inputs de base (énergie, transport, etc.), économisent sur le coût du travail grâce aux paiements en nature et aux arriérés de salaires, développent de même les arriérés fiscaux ${ }^{1}$ et les paiements en nature et ne remplacent pas les biens en capital. La courbe de Phillips se déplace alors vers le bas : ce déplacement correspond à une hausse des arriérés. Au total, la politique monétaire est inefficace car elle ne s'accompagne pas d'un durcissement de la contrainte budgétaire des entreprises.

Les arriérés de salaires permettent aux entreprises de s'immuniser contre la hausse du salaire réel lié à la politique monétaire restrictive. L'emploi reste supérieur à ce qu'il serait si les agents n'étaient pas « myopes » : parce que les salariés anticipent qu'ils seront payés, l'emploi est maintenu à un niveau supérieur à son niveau d'équilibre. Les salariés pensent avoir une chance de recouvrer leurs créances auprès des entreprises parce que celles-ci ne font pas faillite et que l'activité est maintenue. En ne sanctionnant pas les entreprises qui ne paient pas leurs impôts et en effectuant lui-même une partie significative de ses transactions sous la forme de paiements non monétaires (taxes contre services/biens), l'État entretient la myopie des agents (c'est-à-dire, en l'occurrence, leur croyance dans la rentabilité des entreprises et la capacité de ces dernières à payer des salaires élevés) et permet la survie des entreprises. Le déplacement de la courbe de court terme vers le bas correspond donc aussi à l'augmentation des arriérés fiscaux, qui sont une forme de création quasi monétaire selon l'équation (2) de la section précédente.

Si la politique monétaire continue d'être restrictive, l'économie tend à descendre le long de la nouvelle courbe de C vers D et l'État et les salariés doivent à nouveau injecter dans l'économie des crédits sous forme de paiements non monétaires et d'arriérés afin de créer une nouvelle « inflation surprise ». Le processus de compensation par une création quasi monétaire est ainsi itéré tant que la politique monétaire reste restrictive. Ce processus explique la dynamique :

1. d'augmentation limitée du chômage, qui demeure inférieur à son niveau de long terme. Pour avoir une idée de ce niveau d'équilibre, il suffit de songer aux performances en termes d'inflation et de chômage des pays d'Europe centrale qui, après avoir stabilisé l'inflation à un chiffre, ont des taux de chômage significativement plus élevés qu'en Russie. Les travaux réalisés en panel montrent en effet que les taux de croissance ne redeviennent positifs qu'après une période de stabilisation dans laquelle le chômage augmente ${ }^{2}$,

1. Les études montrent que l'État apparaît comme le «prêteur en premier ressort » quand les entreprises ont des problèmes financiers (cf. Ivanova et Wyplosz [1999]).

2. Tichit [2001] montre que le processus de transition implique que le chômage « cause » la croissance. L'absence de restructuration dans les États de la CEI est mise en 
les activités correspondant à l'économie planifiée sont détruites, la restructuration prend place ;

2. de maîtrise apparente de l'inflation officielle. La BERD [1998] souligne cependant le caractère illusoire de la stabilisation macroéconomique affichée en Russie : «From late 1997, the government began to make sharp spending cuts by overhauling budgetary organisations and introducing conditionality on regional transfers. The result was a primary surplus of the federal budget in the first half of 1998, though the continued accumulation of expenditure arrears raised sustainability concerns. » (Rapport sur la transition de la BERD, 1998, p. 187) ;

3. d'augmentation de l'économie virtuelle, à travers les différentes formes de paiements non monétaires.

L'ampleur du phénomène de démonétisation a suscité une littérature abondante, qui complète l'approche que nous avons retenue en mettant l'accent sur :

1. l'accumulation d'arriérés stratégiques : Perotti [1998] ou Schaffer [1998] montrent que le manque de crédibilité du gouvernement favorise les arriérés. Les entreprises anticipent que l'État, tôt ou tard, effacera leurs dettes ou que celles-ci seront érodées par l'inflation, autrement dit que l'État contribue au maintien d'une contrainte budgétaire douce ${ }^{1}$. Pour Hendley et al. [1998], le caractère stratégique des arriérés s'explique par un motif d'évasion fiscale : les taxes n'étant prélevées que quand l'entreprise reçoit un paiement monétaire pour ses livraisons, l'incitation à substituer des moyens de règlement non monétaires est grande.

2. la contrainte de liquidité : dans un contexte où la politique monétaire est fortement restrictive et où le marché du financement est incomplet, les entreprises n'ont pas d'autres solution que de recourir au troc ou de développer des arriérés de paiement, simplement pour survivre (Ellingsen [1998], Linz et Krueger [1998]). Cette approche ne permet toutefois pas de distinguer le troc de court terme et le troc qui correspond à la démonétisation d'une économie qui ne restructure pas.

3. l'insuffisante restructuration de l'économie russe: Gaddy et Ickes [1998], Brana et Maurel [1999] montrent que les entreprises performantes ont moins recours aux paiements non monétaires que les entreprises qui préfèrent investir dans du capital relationnel et éviter les restructurations. Pour Ivanova et Wyplosz [1999], les arriérés s'expliquent par l'existence de pertes sur longue période, liées au manque de restructuration de pans entiers de l'industrie russe, et non à des difficultés passagères.

Les analyses fondées sur la contrainte de liquidité supposent que la politique monétaire restrictive est responsable de la pénurie de liquidités, elles impliquent donc que le développement des paiements non monétaires permet d'éviter une dés-industrialisation massive. On peut au contraire penser, en privilégiant l'hypothèse d'une persistance de la contrainte budgétaire douce, que la politique

perspective avec une dynamique de chômage faible qui se distingue très nettement de celle qui caractérise les transitions réussies, en Europe centrale et orientale.

1. L'accélération des arriérés dans un contexte de stabilisation s'accorde néanmoins mal avec ce type d'analyse (Coricelli et Duchêne [1998]). 
monétaire ne peut pas jouer ce rôle, tout simplement parce qu'elle est inefficace, l'économie réelle réagissant en se soustrayant à l'économie monétaire. Le modèle proposé, fondé sur une analogie entre l'inflation surprise créée par une politique monétaire expansionniste et la myopie des agents qui croient que les arriérés seront payés en cash, propose une synthèse des points 2 et 3 ci-dessus dans laquelle la contrainte de liquidité associée à l'absence de restructuration renforce de manière endogène le phénomène de démonétisation. Il permet en outre d'interpréter dans un cadre macroéconomique simple l'augmentation continue des paiements non monétaires : pour maintenir l'emploi à un niveau supérieur à celui qu'impliquent les possibilités réelles de l'économie, l'État doit sans cesse injecter des crédits dans l'économie. L'absence de restructuration qui en résulte est analogue à l'accélération de l'inflation nécessaire au maintien d'un chômage inférieur au chômage naturel dans une économie de marché : tant que les agents croient que l'économie virtuelle est solvable, qu'ils anticipent que les arriérés de salaires seront payés, l'emploi peut être maintenu à un niveau fictif grâce à une politique fiscale appropriée.

\section{LA RÉSISTANCE DE L'ÉTAT AUX RESTRUCTURATIONS}

L'objectif de la politique monétaire dans les économies en transition est d'abord de stabiliser de manière à rendre possible la sélection des entreprises compétitives. Lorsqu'un processus de restructuration se met en place, l'inflation ne reflète plus le fait que les déficits sont monétisés et correspond aux gains de productivité réalisés. Ce scénario vertueux correspond à ce qui s'est passé en Europe centrale, où l'on a toutefois observé au début du processus de transition le développement d'arriérés de paiements dus au caractère parfois excessivement restrictif de la politique monétaire et à une contrainte de liquidité s'exerçant à l'encontre d'entreprises potentiellement rentables. Cependant, à long terme, il n'y a aucune raison d'établir un lien entre la politique monétaire et les arriérés de paiement. En Russie, en revanche, le développement spectaculaire de ces derniers suggère de s'interroger sur l'existence de relations dynamiques qui mettent en évidence :

1. la substitution au financement monétaire du déficit d'une politique d'augmentation des arriérés fiscaux auprès des administrations publiques. Ce faisant, l'État donne le mauvais exemple et contribue à la généralisation du troc entre les entreprises. L'inefficacité de la politique monétaire a pour conséquence de différer les restructurations, et, à long terme, le chômage augmente malgré l'augmentation des arriérés. Ce scénario est commenté à partir de simples corrélations croisées entre le chômage, l'inflation et les différentes formes d'arriérés ;

2. l'analogie développée avec la courbe de Phillips suggère de compléter l'analyse de la relation de long terme par une analyse de la dynamique de court terme : les relations de coïntégration que nous mettons en évidence montrent qu'à court terme l'injonction de crédits non monétaires permet de maintenir l'emploi à un niveau plus élevé que ce qu'implique l'équilibre, alors qu'à long terme arriérés et chômage augmentent conjointement. 


\section{Corrélations croisées}

Pour Commander et Mumssen [1998], l'augmentation continue des arriérés ne peut s'expliquer que parce qu'il y a une injection de crédit net dans l'économie, sous la forme d'une politique fiscale qui privilégie l'emploi et refuse de sanctionner les entreprises dont la dette fiscale augmente. La responsabilité de l'État dans la démonétisation de l'économie apparaît centrale. Elle se manifeste sous au moins trois formes : l'accumulation d'arriérés fiscaux, le paiement en nature des dettes fiscales et les subventions aux entreprises en difficulté, par l'intermédiaire des entreprises publiques.

Le graphique 2 reflète la forte relation de substitution qui existe entre l'évolution du taux d'inflation (qui peut être le signe du caractère plus ou moins restrictif de la politique monétaire) et l'augmentation des arriérés fiscaux : l'inflation diminue, au prix d'une augmentation significative des arriérés auprès des administrations publiques.

\section{Graphique 2. Corrélation entre arriérés fiscaux et taux d'inflation} (1993-6 ; 1998-1, données mensuelles)

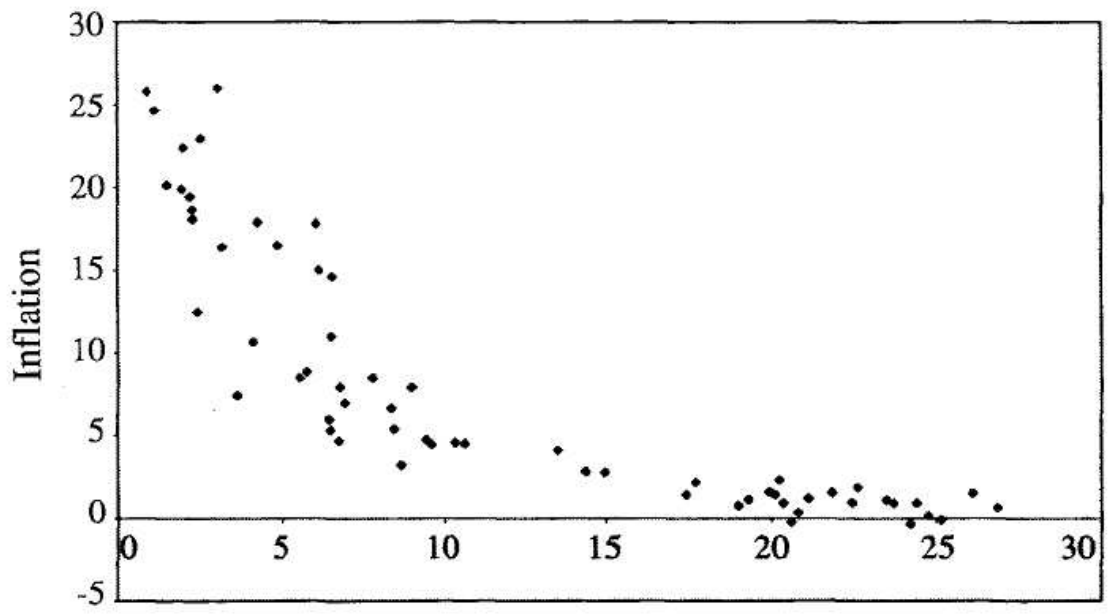

Arriérés auprès des administrations publiques

Source des données: Russian Economic Trend.

Alors que les arriérés de salaires ne semblent pas directement liés aux performances des entreprises, que les crédits interentreprises relèvent d'un fonctionnement normal des relations commerciales, semblables à celui d'une économie de marché, les arriérés fiscaux sont concentrés dans les entreprises en difficultés financières. Schaffer [1998] montre qu'ils sont la source, en Russie, de la contrainte douce de budget des firmes. Alfanderi et Schaffer [1996] indiquent que les 10 à $15 \%$ des entreprises les moins rentables concentraient $44 \%$ des arriérés fiscaux en 1994. De même, Ivanova et Wyplosz [1999] trouvent une relation de causalité entre le pourcentage d'entreprises qui font des pertes et la croissance des arriérés fiscaux. En acceptant l'accumulation de tels arriérés, l'État accorde un financement gratuit à ces firmes ${ }^{1}$, voire une réduction impor-

1. Même si des pénalités dissuasives sont censées s'appliquer. 
tante de leur note fiscale, si le problème des arriérés se résout par le troc ou par des accords de compensation (offsets).

Le lien entre troc et arriérés fiscaux dans le graphique 3 est positif et significatif, suggérant que l'État est au moins en partie responsable de la généralisation du troc. En étant à l'origine d'une partie significative des arriérés de paiement, l'État ne peut que manifester une volonté très faible de recouvrer ses créances. Les premiers sont donc implicitement échangés contre les secondes sous forme de troc: taxes contre biens/services fournis aux administrations publiques.

En acceptant le paiement en nature des dettes fiscales, l'État accorde des réductions fiscales importantes. Ces paiements fiscaux non monétaires représenteraient $40 \%$ des revenus fédéraux et plus de la moitié des budgets de province (Commander et Mumssen [1998]), ce qui correspond à d'importantes subventions implicites. Le mécanisme des Tax offsets consiste à éliminer les obligations fiscales des entreprises en échange de biens et services délivrés au gouvernement. C'est le moyen, pour l'État, de contourner les plafonds de dépenses fixés par le FMI, tandis que l'entreprise qui paie ainsi ses impôts avec des produits invendables aux prix de marché, bénéficie finalement d'une réduction de sa facture fiscale.

\section{Graphique 3. Corrélation entre troc (en \% des ventes industrielles) et arriérés auprès des administrations publiques (1993-6 ; 1998-1, données mensuelles)}

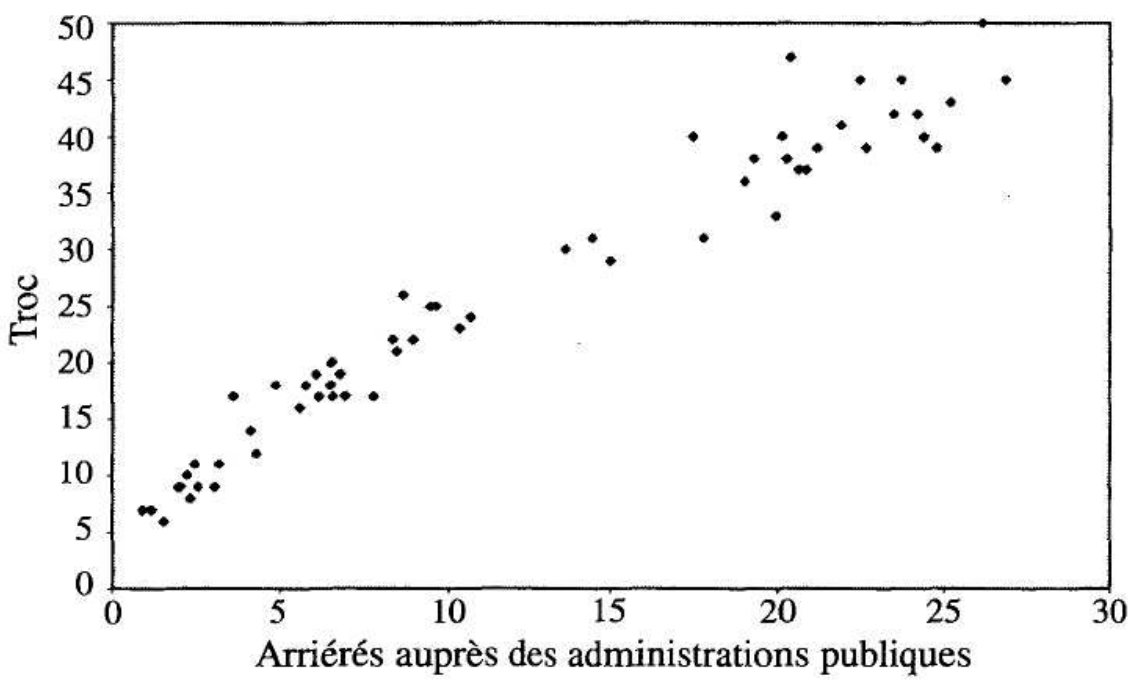

Source des données : Russian Economic Trend, Russian Economic Barometer.

L'État contribue enfin à l'économie virtuelle en accordant des subventions implicites via les entreprises publiques et notamment les monopoles naturels. La décomposition sectorielle des paiements «à recevoir» (Russian Economic Trend, octobre 1997) montre que les secteurs de l'Énergie et des Transports arrivent en tête. Or, ces secteurs sont dominés par des entreprises majoritairement publiques, qui fournissent ainsi, en laissant s'accumuler les arriérés, des biens et services subventionnés. Notamment, les trois monopoles publics que sont Gazprom pour le gaz, RAO UES pour l'électricité et MPS pour les chemins 
de fer, firmes représentant $25 \%$ des ressources fédérales en 1999, ne reçoivent que $10 \%$ de leurs revenus en cash.

L'étude du cas d'une entreprise produisant du charbon par Gaddy et Ickes [1999] permet de bien cerner comment fonctionne l'économie virtuelle. Cette entreprise déclare un montant de ventes pour une valeur de 551,1 milliards de roubles. Cependant en pratique, seul 1 milliard est payé en liquide, tandis que 335 sont reçus sous forme de veksels (titres de dette) et 215 en biens (troc). Les veksels peuvent être revendus avec une ristourne de $80 \%$, les biens avec une ristourne de $56,5 \%$. Au total, la valeur «monétaire » des ventes n'est que de 161,5 milliards de roubles. La démonétisation de l'économie a donc permis à l'entreprise de valoriser « virtuellement » $(3,4$ fois $)$ sa production. Mais le plus intéressant est que les utilisateurs ont obtenu un bien intermédiaire à un prix bien plus faible que celui payé en apparence. Ils apparaissent donc subventionnés. Une des conséquences est qu'il est impossible d'évaluer les performances et la rentabilité des entreprises.

Les relations de troc et l'accumulation d'impayés permettent aux entreprises de diminuer leurs coûts de production, de maintenir un certain niveau de production et d'occuper la main-d'œuvre en excès. Le graphique 4 atteste de la relation positive et significative qui existe à long terme entre le chômage et les arriérés fiscaux, conformément à la dynamique suggérée dans le modèle de la section 1.

\section{Graphique 4. Corrélation entre taux de chômage et arriérés fiscaux (1993-6 ; 1998-1, données mensuelles)}

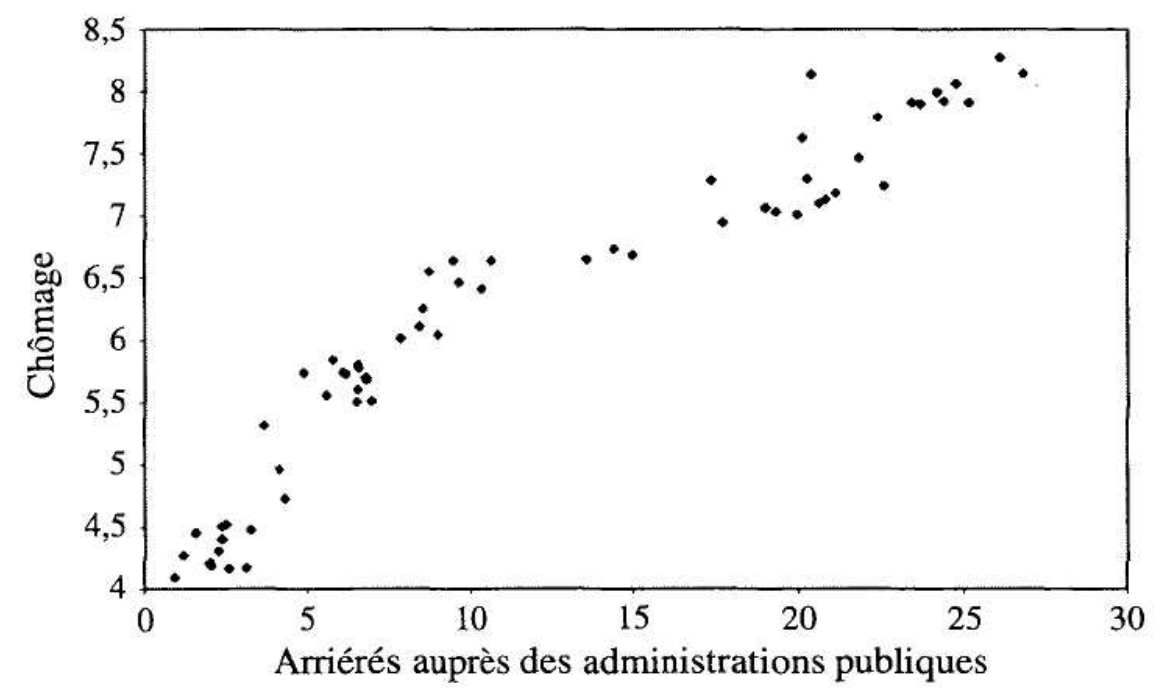

Source des données : Russian Economic Trend.

\section{Relations de coïntégration}

L'augmentation du chômage atteste que l'augmentation de la masse monétaire augmentée par les arriérés ne correspond pas à des perspectives de croissance réelles, mais bien à de l'inflation cachée. L'économie converge vers son niveau de chômage d'équilibre, mais, à court terme, les agents (privés et publics) privilégient le maintien de l'activité et de l'emploi en injectant des crédits dans l'économie, c'est-à-dire en accumulant des impayés. Cet arbitrage est mis en 
évidence grâce à des relations de coïntégration entre arriérés, troc, inflation et chômage.

Tableau 2. Tests de coïntégration pour les arriérés de salaires

$(1993-3 ; 1998-11)$

\begin{tabular}{ccccc}
\hline \multicolumn{5}{c}{ Rang de la matrice $\mathrm{P}_{0}$} \\
\hline $\mathrm{H}_{0}$ : rang = $\mathrm{p}$ & $\begin{array}{c}\text { Statistique de la valeur } \\
\text { propre maximale }\end{array}$ & Seuil à 95\% & $\begin{array}{c}\text { Statistique de la } \\
\text { Trace }\end{array}$ & Seuil à 95\% \\
\hline $\mathrm{p}=0$ & $34,59^{* *}$ & 27,1 & $54,9 * *$ & 47,2 \\
$\mathrm{p}<=1$ & 13,21 & 21 & 20,31 & 29,7 \\
$\mathrm{p}<=2$ & 7,056 & 14,1 & 7,094 & 15,4 \\
$\mathrm{p}<=3$ & 0,038 & 3,8 & 0,03812 & 3,8 \\
\hline
\end{tabular}

Vecteurs coïntégrants

Arriérés de salaires : Arrs* Taux de chômage : Tch Troc : Troc* Taux d'inflation : Inf

\begin{tabular}{cccl}
1 & $-0,057$ & $-1,095$ & 0,2452 \\
$-2,9$ & 1 & 0,1006 & 0,1902 \\
$-138,7$ & 21,67 & 1 & 0,8919 \\
5,247 & 7,576 & 0,3982 & 1 \\
\hline
\end{tabular}

* Les arriérés sont exprimés en pourcentage des ventes.

Les variables étant stationnaires en différence, on utilise la méthode du maximum de vraisemblance de Johansen pour déceler l'existence de vecteurs cointégrants. Le nombre de retards (5) a été choisi sur la base du critère d'information de Hannan-Quinn. Les données sont mensuelles.

* : significatif à $95 \%$; ** : significatif à $99 \%$. $\mathrm{F}(80,179)=19,172 * *$

Sources des données : Russian Economic Trend, Russian Economic Barometer.

Le tableau 2 indique l'existence d'un seul vecteur coöntégrant. La relation de long terme associée s'écrit :

$$
\text { Arrs }=0,057 \text { Tch }+1,095 \text { Troc }-0,2452 \operatorname{Inf}
$$

Ainsi, les arriérés de salaires sont associés à long terme à :

1. une augmentation du chômage. À long terme, le chômage augmente vers son niveau d'équilibre malgré l'augmentation des arriérés de salaires qui permet d'arbitrer à court terme en faveur d'un niveau de chômage inférieur ${ }^{1}$;

2. une diminution du taux d'inflation. Le signe négatif et significatif de la variable Inf montre très clairement que les arriérés augmentent quand la politique monétaire se durcit. Quand l'inflation diminue, le coût réel du

1. On peut expliquer également le signe positif de la variable Taux de chômage Tch en remarquant que le pouvoir de négociation des salariés touchés par les arriérés de salaire diminue lorsque le niveau du chômage augmente. 
travail augmente ce qui incite les entreprises à neutraliser cette hausse en développant les impayés ${ }^{1}$;

3. une augmentation du troc. Le lien entre arriérés et relations de troc est double. D'une part, l'importance du troc réduit les disponibilités monétaires des entreprises leur permettant d'effectuer les paiements de salaires, ce qui favorise les arriérés. D'autre part, une part importante du troc est liée à des compensations de dette.

Tableau 3. Tests de coïntégration pour les arriérés fiscaux

$(1993-6 ; 1998-1)$

\begin{tabular}{ccccc}
\hline \multicolumn{5}{c}{ Rang de la matrice $\mathrm{P}_{0}$} \\
\hline $\mathrm{H}_{0}$ : rang = p & $\begin{array}{c}\text { Statistique de la valeur } \\
\text { propre maximale }\end{array}$ & Seuil à $95 \%$ & $\begin{array}{c}\text { Statistique de la } \\
\text { Trace }\end{array}$ & Seuil à $95 \%$ \\
\hline $\mathrm{p}=0$ & $28,95^{*}$ & 27,1 & $51,82^{*}$ & 47,2472 \\
$\mathrm{p}<=1$ & 16,87 & 21 & 22,87 & 29,7 \\
$\mathrm{p}<=2$ & 5,859 & 14,1 & 6,002 & 15,4 \\
$\mathrm{p}<=3$ & 0,1426 & 3,8 & 0,1426 & 3,8 \\
\hline
\end{tabular}

\begin{tabular}{cccc}
\hline \multicolumn{4}{c}{ Vecteurs cö̈ntégrants } \\
\hline Arriérés fiscaux : Arrf & Taux de chômage : Tch & Troc : Troc & Taux d'inflation : Inf \\
\hline 1 & $-0,9654$ & $-32,41$ & 2,374 \\
$-0,4401$ & 1 & 6,204 & 13,35 \\
0,1041 & 0,05649 & 1 & 1,184 \\
$-0,4316$ & 0,3744 & $-0,6195$ & 1 \\
\hline
\end{tabular}

Le nombre de retards (5) a été choisi sur la base du critère d'information de Hannan-Quinn.

* : significatif à $95 \%$; ** : significatif à $99 \% . F(80,128)=15,009 * *$

Sources des données : Russian Economic Trend, Russian Economic Barometer.

Le tableau 3 indique l'existence d'un seul vecteur coïntégrant. La relation de long terme associée s'écrit :

$$
\text { Arrf }=0,965 \text { Tch }+32,41 \text { Troc }-2,374 \operatorname{Inf}
$$

Ainsi, à long terme, on retrouve les relations mises en évidence à partir de simples corrélations. Les arriérés fiscaux augmentent quand le chômage augmente, l'inflation diminue et le troc se développe.

Les modèles à correction d'erreurs (tableau 4) confirment l'existence d'un arbitrage à court terme entre chômage et arriérés; les arriérés (fiscaux et de salaires) sont négativement corrélés au taux de chômage. La démonétisation apparaît donc bien comme le moyen de maintenir l'emploi, mais seulement à

1. En outre, l'augmentation des taux d'intérêt incite les entreprises à substituer au financement monétaire le crédit « gratuit » que constituent les arriérés, notamment salariaux. 
Tableau 4. Modèles à correction d'erreurs associés aux relations (3) et (4)

\begin{tabular}{|c|c|c|c|c|c|}
\hline \multicolumn{3}{|c|}{$\begin{array}{c}\text { Arriérés de salaires ( } \square \text { Arrs) } \\
\text { 1993(4)-1998(11) }\end{array}$} & \multicolumn{3}{|c|}{$\begin{array}{c}\text { Arriérés fiscaux (ㄱArrf) } \\
\text { 1993(7)-1998(1) }\end{array}$} \\
\hline Variables & Coefficient & $\begin{array}{l}\text { Statistique t de } \\
\text { Student }\end{array}$ & Variables & Coefficient & $\begin{array}{l}\text { Statistique t de } \\
\text { Student }\end{array}$ \\
\hline Constante & 0,0003 & 1,534 & Constante & 0,3069 & 1,112 \\
\hline$\square$ Arrs-2 & $-0,351$ & $-2,973$ & $\square A r r f-1$ & 0,7508 & 3,718 \\
\hline$\square$ Arrs-6 & $-0,352$ & $-2,633$ & $\square A r r f-4$ & $-0,4719$ & $-2,999$ \\
\hline$\square$ Tch-1 & $-0,0022$ & $-2,448$ & $\square T c h-2$ & $-0,1822$ & $-2,449$ \\
\hline$\square$ Troc & 0,0031 & 2,566 & $\square$ Troc & 0,0253 & 2,702 \\
\hline$\square$ Troc-1 & $-0,0157$ & $-2,710$ & $\square$ Troc-2 & 0,0397 & 3,595 \\
\hline$\square$ Troc-2 & 0,0032 & 2,746 & $E C M-1$ & $-0,6767$ & $-2,797$ \\
\hline$\square$ Troc-3 & 0,0025 & 2,335 & & & \\
\hline$\square \operatorname{lnf}$ & $-0,0105$ & $-6,879$ & & & \\
\hline$\square I n f-2$ & $-0,0036$ & $-1,633$ & & & \\
\hline$\square I n f-3$ & 0,009 & 2,154 & & & \\
\hline ECM-1 & $-0,015$ & $-2,811$ & & & \\
\hline \multicolumn{3}{|c|}{$\mathrm{R}^{2}=0,596 \mathrm{DW}=1,81$} & \multicolumn{3}{|c|}{$R^{2}=0,608 \quad D W=2,15$} \\
\hline \multicolumn{3}{|c|}{ AR $: F(5,51)=0,247$ ARCH : $F(5,46)=0,492$} & \multicolumn{3}{|c|}{ AR $: F(4,44)=0,575$ ARCH $: F(4,40)=0,141$} \\
\hline
\end{tabular}

Avec $E C M$, le terme à correction d'erreurs tiré des relations de long terme. Toutes les variables, sauf le terme à correction d'erreurs, sont en différences. Les données sont mensuelles.

Sources des données : Russian Economic Trend, Russian Economic Barometer.

court terme. À moyen terme en effet, le taux de chômage converge vers son niveau d'équilibre de long terme et augmente pour un niveau croissant d'arriérés.

L'économie virtuelle ne peut survivre que parce qu'il y a injection continue de financements nets dans l'économie. Cette injection provient de l'État, des salariés et des entreprises à valeur ajoutée positive. L'État a intérêt au maintien de l'économie virtuelle pour des raisons politiques, liées notamment à l'emploi et aux services sociaux offerts par les entreprises, et parce que subsistent des liens étroits entre l'État et les dirigeants de certaines entreprises. Les salariés acceptent les arriérés de salaires ou les paiements en nature car ils anticipent une amélioration de la situation économique. Ils préfèrent en outre rester au sein de l'entreprise car ils bénéficient de ses services sociaux alors que, compte tenu du marché du travail, ils ne seraient pas sûrs de trouver mieux ailleurs. Pour Brown [1998], les salariés acceptent la baisse de leur rémunération via les impayés en échange de licenciements réduits. Cette théorie d'ajustement du marché du travail est plausible dans le cas d'entreprises dont l'objectif est le paternalisme et l'attrait de subventions publiques. Toutes choses égales par ailleurs, plus l'entreprise a un nombre important de salariés et plus le volume d'arriérés de salaires est important, plus elle est susceptible de recevoir des subventions publiques. Enfin, les entreprises profitables acceptent un transfert de valeur vers les entreprises en situation de détresse financière car l'État leur garantit en échange un certain nombre d'avantages : conservation d'une part importante des profits, maintien d'une situation de monopole, accès au secteur exportateur plus rentable, etc. 


\section{LES IMPLICATIONS POLITIQUES DE L'ÉCONOMIE VIRTUELLE}

\section{Faut-il éradiquer l'économie virtuelle ?}

L'économie virtuelle coûte cher: le chômage augmente malgré tout, mais l'absence de restructuration implique que la reprise de la croissance est différée. Les travaux du FMI montrent que, sans stabilisation, il n'y a pas de reprise de la croissance et que le coût de la transition est plus élevé pour les pays qui stabilisent lentement et dans lesquels le chômage augmente progressivement. La spécificité de la Russie est qu'une fausse stabilisation s'est produite.

L'économie virtuelle réduit également l'efficacité des réformes. Les entreprise sont en effet immunisées contre les politiques restrictives et contre les politiques de libéralisation, parce que l'économie virtuelle a empêché le durcissement des contraintes budgétaires. Dans l'économie virtuelle, les entreprises peuvent continuer à produire des biens qui ne pourraient être vendus sur un marché, car ces biens peuvent être utilisés pour le troc ou pour les paiements fiscaux ou salariaux (Gaddy et Ickes [1999]).

L'économie virtuelle affaiblit les institutions, ce qui retarde la stabilisation et la restructuration. La démonétisation est à la fois la conséquence et la cause de 1'affaiblissement des institutions (Hendley et al. [1998]).

Par exemple, la faiblesse du système fiscal (taux et pénalités excessifs) est une incitation au développement des substituts non monétaires. Celui-ci affaiblit à son tour la collecte fiscale (directement à travers l'évasion fiscale et indirectement en réduisant l'assiette fiscale) et la rend plus difficile en rendant opaques les performances des entreprises. Les arriérés fiscaux impliquent que l'Etat n'a pas les moyens de financer un système de redistribution qui permettrait d'indemniser les perdants de la restructuration. Il est donc incité à accepter le développement des arriérés, comme condition de maintien de l'emploi au sein des entreprises. Cette situation est créatrice d'inégalités qui atteignent les plus vulnérables : les retraités (Kolev et Saget [2001]) et les salariés dont les compétences spécifiques ne leur permettent pas de quitter l'entreprise, même lorsqu'elle est vouée à disparaître (Pailhé et Pascal [2001], Koumakhov et Najman [2001]). Elle est créatrice également de mécontentement : on montre notamment que le bien-être des individus est négativement corrélé à l'existence d'arriérés, et que si le bien-être atteint dans une situation de chômage est inférieur à celui atteint par un salarié, la situation d'un salarié avec arriéré est moins bonne que celle d'un inactif (Clark et Maurel [2001]).

De même, le processus de démonétisation est doublement lié à la politique monétaire. D'un côté, comme nous l'avons montré dans la première section, le développement des monnaies privées complique la conduite de la politique monétaire en affaiblissant le contrôle monétaire de l'économie par les autorités. Son efficacité étant réduite, la politique monétaire doit devenir plus restrictive, ce qui est un facteur incitatif supplémentaire à la démonétisation. Dans ce contexte, la politique monétaire restrictive crée une contrainte de liquidité qui est particulièrement préjudiciables aux entreprises potentiellement rentables. Celles-ci, ne pouvant s'adresser aux banques compte tenu des imperfections du marché du crédit, sont à leur tour obligées d'avoir recours aux moyens de paiement non monétaires (Brana et Maurel [1999]). 
La démonétisation de l'économie affaiblit enfin la plupart des institutions en rendant opaque la situation financière des entreprises : banques et autres institutions financières, bourse, etc.

\section{La politique monétaire peut-elle être utile?}

La politique monétaire est non seulement devenue inefficace, mais elle contribue indirectement au développement de l'économie virtuelle. Deux solutions sont possibles : la première consiste à inclure les arriérés de paiement dans la définition de la cible monétaire et à imposer une politique monétaire restrictive à partir de cette mesure élargie de la monnaie. Mais cette solution apparaît risquée, compte tenu des difficultés de contrôle qui ne manqueraient pas de se poser aux autorités. En outre, cette solution ne résout en rien les raisons de la démonétisation croissante de l'économie.

L'alternative est d'envisager une politique monétaire moins restrictive qui, à défaut de résoudre le problème des entreprises en situation de détresse financière, pourrait, en théorie, réduire le poids de la contrainte de liquidité pesant sur les entreprises les plus profitables. Une injection monétaire additionnelle n'a cependant aucune chance d'aller vers ces entreprises. Les firmes bancaires ne sont pas capables de discriminer leurs emprunteurs ${ }^{1}$, ce qui les conduit à refinancer passivement leurs débiteurs ou à développer des activités plus profitables. La création monétaire aurait toutes les chances d'être directement investie en devises, plus rentables, sans beaucoup affecter l'économie domestique. Elle ne reviendrait finalement qu'à différer la restructuration et l'inflation risquerait d'ajuster instantanément l'écart entre la croissance réelle et la quantité de monnaie réelle mise en circulation.

La probabilité d'un échec est d'autant plus forte qu'aujourd'hui l'économie virtuelle est très bien organisée et que l'économie monétaire, en particulier les systèmes financier et bancaire, ont perdu toute leur crédibilité. Il faut donc mettre en place les incitations qui encourageront les entreprises à réintégrer l'économie monétaire.

\section{Quelles directions, concrètement?}

Restaurer la crédibilité de l'État. Une réforme fiscale doit être entreprise visant à simplifier le système, à réduire les taux d'imposition et à supprimer les nombreuses exemptions. Un impôt juste a davantage de chances d'être perçu, et ce, d'autant plus que l'État respecte lui-même les règles du jeu en payant ses fournisseurs. Si l'Etat choisit de maintenir des subventions, il doit le faire dans la transparence.

Activer les institutions de marché. Les entreprises non rentables font du troc car les prix non monétaires sont supérieurs aux prix mondiaux. Pour ces entreprises, la démonétisation correspond à une stratégie de survie, dans un contexte normal elles devraient faire faillite. Il faut donc renforcer les droits de pro-

1. Cf. Brana, Maurel et Sgard [1999]. 
priété ${ }^{1}$, les procédures de faillites, faciliter la concurrence, l'entrée de nouvelles entreprises et réduire le degré de concentration du marché ${ }^{2}$. La remonétisation passe ainsi paradoxalement par le maintien d'une politique monétaire restrictive, qui ne peut être efficace que si les institutions de marché sont activées.

Instaurer une intermédiation bancaire. La politique monétaire restrictive suppose la mise en place préalable d'un système bancaire compétent, capable de sélectionner les bonnes entreprises qui ne devraient pas être affectées par la contrainte de liquidité. Pour cela, une possibilité est de faciliter l'entrée des banques étrangères et de clarifier les fonctions des banques. Celles-ci ne doivent notamment plus être chargées de l'obligation de collecte fiscale qui est un facteur important de désintermédiation.

\section{CONCLUSION}

La démonétisation de l'économie russe peut s'expliquer, dans le cadre connu du modèle d'arbitrage de Phillips, à partir de l'analogie entre inflation non anticipée par les agents et augmentation des paiements non monétaires, c'està-dire augmentation de l'inflation cachée. La politique monétaire russe n'apparaît plus comme restrictive, dès lors que l'on prend en compte sa composante non monétaire et que l'on analyse la progression de l'économie virtuelle comme un arbitrage des autorités en faveur de l'emploi, préjudiciable à la stabilisation macroéconomique.

Un modèle très simple est testé à partir de données macroéconomiques du Russian Economic Trend, du Russian Economic Barometer, et du Goskomstat. Les arriérés apparaissent très significativement corrélés aux variables de la politique économique, c'est-à-dire l'inflation et le chômage. Si, dans le cadre d'une politique monétaire restrictive, la maîtrise de l'inflation faisait disparaître les entreprises non rentables, le montant agrégé des arriérés diminuerait et il n'y aurait pas de lien, à long terme, entre les arriérés et les instruments de la politique économique. Or des relations de long terme sont mises en évidence : l'augmentation des paiements non monétaires permet de maintenir le chômage à un niveau qui, bien que croissant, demeure inférieur au niveau d'équilibre, l'inflation étant compensée par l'augmentation des arriérés. La maîtrise de l'inflation n'est donc qu'illusoire et entretient un phénomène de myopie à court terme des agents qui est tout à fait analogue à la «pathologie » décrite par les monétaristes.

Les implications sur le type de politique économique à mettre en œuvre sont les suivantes: la politique monétaire restrictive, puisque son objectif est de contribuer à la sélection des entreprises compétitives, doit être maintenue. Cette sélection opère à travers l'augmentation des taux d'intérêt et l'existence d'un

1. Le lien entre droit de propriété et troc est explicitement modélisé dans Guriev, Makarov, et Maurel [2000].

2. L'augmentation du troc est plus forte dans les segments de marché caractérisés par un fort degré de concentration (Guriev et Kvasov [1999]). 
système bancaire efficace : la réforme de ce système est donc prioritaire, un moyen d'améliorer les performances des banques étant d'importer le savoirfaire occidental. Enfin, il serait sans doute souhaitable que l'exotisme dans lequel l'État finance ses subventions au moyen du troc et en accumulant des créances auprès de grosses entreprises soit remplacé par une gestion transparente et démocratique des comptes publics...

\section{RÉFÉRENCES BIBLIOGRAPHIQUES}

ALFANDARI G., SCHAFFER M.E. [1996], « "Arrears" » in the Russian Enterprise Sector », dans COMMANDER S., FAN, SCHAFFER M.E. (eds.), Enterprise Restructuring and Economic Policy in Russia, EDI Development Studies, Washington DC, The World Bank.

BERD [1998], Rapport sur la Transition.

Brana S., MaURel M., SGARD J. [1999], « Enterprise Adjustment and the Role of Bank Credit in Russia : Evidence from a 420 Firm's Qualitative Survey », Comparative Economic Studies, 42 (4), p. 47-69.

Brana S., MAUREL M. [1999], «Barter in Russia : Liquidity shortage versus Lack of Restructuring », CEPR Discussion Paper, 2258.

BROWN J.D. [1998], «Infrequent Bankruptcy asymmetric information, and excess labor in the Russian economy », SITE Working Paper, 135.

Clark A., Maurel M. [2001], « Well-Seing and Wage Arrows in Russian Panel Data ", HSE Economic Journal, à paraître.

COMMANDER S., MumSSEN C. [1998], « Understanding Barter in Russia », EBRD Working Paper, 37.

CORICELLI F., DUCHÊNE G. [1998], «Inter-enterprise arrears in Ukraine : Causes, characteristics and Policy Implications », Mimeo.

Ellingsen T. [1998], «Payments in Kind », Working Paper Series in Economics and Finance, 244 (Stockholm School of Economics).

ERICSSON R.E., ICKES B.W. [1999], "A Model of Russia's "Virtual Economy" », Mimeo.

GADDY C., ICKES B.W. [1998], « To Restructure or Not to Restructure : Informal Activities and Enterprise Behavior in Transition », The Davidson Institute Working Paper Series, 134.

GADDY C., ICKES B.W. [1999], Russia's Virtual Economy, Brookings Institution, à paraître.

Guriev S., Kvasov D. [1999], « Barter in Russia : the Role of Market Power », RECEP Working Paper, 3.

Guriev S., MaKarov I., Maurel M. [2000], « Debt Overhang and Barter in Russia », CEPR Working Paper (à paraître).

HENDLEY K., ICKes B.W., RYTERMAN R. [1998], « Remonetizing the Russian Economy », dans Broadman H.G. (ed.), Russian Enterprise Reform : Policies to Further the Transition, The World Bank, Washington DC, novembre.

IVAnova N., WypLOSZ C. [1999], « Arrears : The Tide that is Drowning Russia », RECEP Working Paper Series, 1. 
JOHANSEN S. [1995], Likelihood-based Inference in Cointegrated Vector Autoregressive Models, Oxford, Oxford University Press.

Kolev A., SAGET C. [2001], « Travail et Retraite en Russie : 1992-96, le rôle des arriérés de pension », Économie et Statistique (à paraître).

KoumaKHOV R., NAJMAN B. [2001], «La question des sureffectifs en Russie : une explication en termes de compétences ", Revue économique, ce numéro.

LINZ S.J., KRUEGER G. [1998], « Enterprise Restructuring in Russia's Transition Economy : Formal and Informal Mechanisms », Comparative Economic Studies, 40 (2), été, p. 5-52.

PAILHE A., PASCAL A. [2001], « Arriérés de salaire, congés obligatoires et participation au marché du travail russe », Revue économique, ce numéro.

PEROTTI E.C. [1998], « Inertial Credit and Opportunistic arrears in transition », European Economic Review, 42, p. 1703-1725.

SCHAFFER M.E. [1998], « Do Firms in Transition Economies Have Soft Budget Constraints ? A Reconsideration of Concepts and Evidence ", Journal of Comparative Economics, 26, p. 80-103.

TICHIT A [2001], «Analyse des divergences de chômage entre les pays en transition post-communistes », Revue d'études comparatives Est-Ouest (à paraître). 